\title{
Knockdown of GBAS regulates esophageal cancer cell viability and apoptosis
}

\author{
JUN PENG, KE MA, HAO RONG, BO XIAO, JIANG ZHU and JIN-TAO HE

\begin{abstract}
Department of Thoracic Surgery, Sichuan Cancer Hospital and Institute, Sichuan Cancer Center, School of Medicine, University of Electronic Science and Technology of China, Chengdu, Sichuan 610054, P.R. China
\end{abstract}

Received September 28, 2020; Accepted April 22, 2021

DOI: $10.3892 / \mathrm{mmr} .2021 .12162$

\begin{abstract}
Esophageal cancer (EC) is the sixth leading cause of cancer-related mortality worldwide, with the incidence gradually increasing each year. Therefore, further clarifying the mechanism underlying the development of EC may be beneficial for identifying novel biomarkers and targets for its treatment. The present study aimed to determine the functional roles of glioblastoma-amplified sequence (GBAS), a newly identified gene that has been reported to play crucial roles in multiple types of cancer, including in the malignant behavior of EC cells, such as cell viability, colony formation, cell apoptosis and cell cycle progression. The results of the present study revealed that, in vitro, the knockdown of GBAS significantly suppressed cell viability and colony formation in TE-1 and KYSE-150 cell lines, using a Celigo cell count analysis and colony formation assay respectively, whereas the apoptotic rate of EC cells was significantly increased by the knockdown of GBAS using Annexin V APC staining. Furthermore, following GBAS knockdown, the cell cycle progression of TE-1 and KYSE-150 cells was arrested in the $\mathrm{G}_{1}$ phase using PI staining. In conclusion, the findings of the present study suggested that GBAS may serve a role in EC by regulating cell viability, apoptosis and cell cycle progression.
\end{abstract}

\section{Introduction}

Esophageal cancer (EC) is one of the most common types of gastrointestinal cancer worldwide, which is accompanied by a poor prognosis and unsatisfactory therapeutic outcomes $(1,2)$. According to 2015 statistics, 245,651 new cases of EC were diagnosed in China; due to the high incidence and mortality

Correspondence to: Professor Jin-Tao He, Department of Thoracic Surgery, Sichuan Cancer Hospital and Institute, Sichuan Cancer Center, School of Medicine, University of Electronic Science and Technology of China, 55 South Renmin Road, Chengdu, Sichuan 610054, P.R. China

E-mail: hjtabc3@163.com

Key words: esophageal cancer, glioblastoma-amplified sequence, viability, apoptosis, cell cycle rates of 17.87/100,000 and 13.68/100,000 individuals, respectively (3), EC poses a serious health and socioeconomic burden in China. In total, $>95 \%$ of EC cases are defined as squamous cell carcinomas or adenocarcinomas (3). EC is often asymptomatic during the early stages and, therefore, the majority of cases are diagnosed at an advanced stage (4). To date, there has been a lack of specific prevention strategies for $\mathrm{EC}$, particularly in patients with distant metastases $(5,6)$. Thus, it is urgent to identify novel molecular biomarkers and targets for the early screening and treatment of this malignancy.

Glioblastoma-amplified sequence (GBAS), also known as nipsnap homolog 2 (NIPSNAP), belongs to the NIPSNAP family, and is located in the vicinity of marker D7S499 on chromosome 7p12 (7). GBAS encodes a mitochondrial protein that contains tyrosine phosphorylation sites, transmembrane motifs and a mitochondrial targeting sequence (8), which is present on the surface of mitochondria (9). Emerging evidence has indicated the functional roles of GBAS in cellular activities. For example, Abudu et al (10) reported that NIPSNAP family members, including NIPSNAP1 and NIPSNAP2, serve crucial roles in mitophagy by recruiting selective autophagy-related proteins (10). Multiple previous studies have also suggested that GBAS may be involved in the initiation, development and progression of several types of solid tumors alongside the co-amplification with other cellular genes, such as EGFR, which is an important oncogene in several cancer types (8). For example, a previous study revealed that GBAS was co-amplified with EGFR, where the EGFR intronic variant was associated with GBAS expression in early-stage non-small cell lung cancer (NSCLC) (11). Another study demonstrated that GBAS expression levels were downregulated in oral squamous cell carcinoma (OSCC) and regulated the viability and apoptosis of cancer cells through activating the p53 signaling pathway (12). Therefore, GBAS may serve as a molecular biomarker of tumorigenesis; however, the mechanisms underlying the role of GBAS in the pathogenesis of EC remain poorly understood.

The present study aimed to perform a comprehensive analysis of the function of GBAS as a potential important oncogene in the development of EC. The effects of GBAS knockdown on the viability, colony formation ability, cell cycle progression and apoptosis of EC cells were investigated in vitro, in order to determine whether GBAS may serve as a novel oncogene in the development of EC. 


\section{Materials and methods}

Patient studies. A total of 15 esophageal squamous cell carcinoma (5 female patients and 10 male patients) and matched adjacent tissues ( $5 \mathrm{~cm}$ away from cancer tissue) were obtained at Sichuan Cancer Hospital (Chengdu, China), between May 2019 and April 2020, from the patients before any clinical interventions. The samples were frozen and stored in liquid nitrogen until further use. All the patients included had achieved R0 resection and provided complete clinicopathological data. A total of 12 patients had tumors located in the middle part of the thoracic esophagus. All patients were diagnosed with EC by two pathologists. The protocols of the present study were approved by the Ethics Committee of Sichuan Cancer Hospital (approval no. SCCHEC-02-2017-043) and written informed consent was obtained from all patients prior to participation.

Cell lines and culture. Human esophageal epithelial cells (HEEC) and human EC cell lines (TE-1, KYSE-150, EC9706 and ECA-109) were obtained from the American Type Culture Collection. 293T cells were purchased from The Cell Bank of Type Culture Collection of The Chinese Academy of Sciences. The HEEC and EC cell lines were cultured in RPMI-1640 medium (Invitrogen; Thermo Fisher Scientific, Inc.), while the 293T cell was cultured in DMEM medium (Invitrogen; Thermo Fisher Scientific, Inc.), supplemented with 10\% FBS (Gibco; Thermo Fisher Scientific, Inc.), $100 \mathrm{U} / \mathrm{ml}$ penicillin and $100 \mathrm{~g} / \mathrm{ml}$ streptomycin, and maintained in a humidified atmosphere with $5 \% \mathrm{CO}_{2}$ at $37^{\circ} \mathrm{C}$.

Lentiviral-mediated GBAS knockdown. The package of lentivirus was used 3rd generation system. The GV115 (pGCSIL-GFP; Shanghai GeneChem Co., Ltd.) lentivirus was used for constructing GBAS-knockdown vectors. The short hairpin RNA (shRNA/sh) sequence targeting GBAS used was as follows: Forward, 5'-CCGGCCATGTGAAAGTCCTGTT GAACTCGAGTTCAACAGGACTTTCACATGGTTTTTG-3' and reverse, 5'-AATTCAAAA ACCATGTGA AAGTC CTGTTGAACTCGAGTTCAACAGGACTTTCACATGG-3'. After cloning the shGBAS or shCtrl sequence into the GV115 vectors, the lentivirus construction system, including the GV115-GBAS vector $(20 \mu \mathrm{g})$, and Helper $1.0(15 \mu \mathrm{g}$; Shanghai GeneChem Co., Ltd.) and Helper 2.0 (10 $\mu \mathrm{g}$; Shanghai GeneChem Co., Ltd.) packaging vectors, were co-transfected into 293T cells using Lipofectamine ${ }^{\circledR} 2000$ (Invitrogen; Thermo Fisher Scientific, Inc.). Following 72 h of transfection, the freshly harvested virus supernatants were collected by ultracentrifugation $\left(25,000 \mathrm{xg}\right.$ at $4^{\circ} \mathrm{C}$ for $\left.2 \mathrm{~h}\right)$ and subsequently stored at $-80^{\circ} \mathrm{C}$. The multiplicity of infection for EC cell transfection was 20 . After $72 \mathrm{~h}$, the transfection efficiency of GBAS knockdown (shGBAS) was determined using reverse transcription-quantitative PCR (RT-qPCR) and western blotting.

$R T-q P C R$. HEEC, EC9760, ECA-109, KYSE-150 and TE-1 cells were washed with PBS three times and total RNA was extracted using TRIzol ${ }^{\circledR}$ reagent (Invitrogen; Thermo Fisher Scientific, Inc.), according to the manufacturer's protocol. Total RNA was reverse-transcribed into cDNA using a reverse-transcribed kit according to the manufacturer's protocol (Promega Corporation).
qPCR was subsequently performed using SYBR Green Master mix (Takara Bio, Inc.). The following thermocycling conditions were used for qPCR: Initial denaturation at $95^{\circ} \mathrm{C}$ for $5 \mathrm{~min}$; followed by 40 cycles at $95^{\circ} \mathrm{C}$ for $15 \mathrm{sec}, 60^{\circ} \mathrm{C}$ for $30 \mathrm{sec}$ and $70^{\circ} \mathrm{C}$ for $10 \mathrm{sec}$. Relative expression levels were calculated using the $2^{-\Delta \Delta C \mathrm{C}}$ method (13). The following primer sequences were used for qPCR: GBAS forward, 5'-TTCGTAAGGCAAGAAGTGAC-3' and reverse, 5'-GTCGGAGTTGGTAAGACCTG-3'; and GAPDH forward, 5'-TGACTTCAACAGCGACACCCA-3' and reverse, 5'-CACCCTGTTGCTGTAGCCAAA-3'. GAPDH was used as the internal reference gene.

Western blotting. KYSE-150 and TE-1 cells were centrifuged at $100 \mathrm{x} \mathrm{g}$ for $5 \mathrm{~min}$ at room temperature and washed with cold PBS twice. The collected cells were resuspended in RIPA lysis buffer (Beyotime Institute of Biotechnology), supplemented with $1 \mathrm{mM}$ PMSF (Sigma-Aldrich; Merck KGaA), and maintained on ice for $30 \mathrm{~min}$. Following centrifugation at $12,000 \mathrm{x} \mathrm{g}$ for $15 \mathrm{~min}$ at $4{ }^{\circ} \mathrm{C}$, the supernatant was transferred into new Eppendorf tubes and protein quantification was performed using a BCA assay (Thermo Fisher Scientific, Inc.). Subsequently, $25 \mu \mathrm{g}$ protein/lane was separated via SDS-PAGE on $12 \%$ gel, then separated proteins were transferred onto PVDF membranes and blocked in 5\% skimmed milk in TBS with $1 \%$ Tween-20 (TBST) for $2 \mathrm{~h}$ at a room temperature. The membranes were incubated with the following primary antibodies at $4^{\circ} \mathrm{C}$ overnight: Anti-GBAS (1:2,000; cat.no. ab153833; Abcam), anti-caspase-3 (1:1,000; cat. no. 9662; CST) and anti-caspase-3 (1:1,000; cat. no. 9661; CST Biological Reagents Co., Ltd.) anti-GAPDH (1:2,000; cat. no. sc-32233; Santa Cruz Biotechnology, Inc.). Following primary antibody incubation, the membranes were incubated with anti-rabbit (cat. no. 7074) and anti-mouse (cat. no. 7076) secondary antibodies (1:2,000; Cell Signaling Technology, Inc.) at room temperature for $2 \mathrm{~h}$. The membranes were washed three times with TBST and protein bands were visualized using enhanced chemiluminescence reagent (Cell Signaling Technology, Inc.).

Cell viability assay. TE-1 and KYSE-150 cells were seeded into 96 -well plates at a cell density of $2.0 \times 10^{3}$ cells/well, with three replicates/group. The cells were subsequently cultured with $100 \mu 1$ RPMI-1640 medium for a total of 5 days. Every $24 \mathrm{~h}$, a Celigo image cytometer system (Nexcelom Bioscience LLC) was used to determine cell number, and the number of cells with green fluorescence in each well was calculated using a Celigo cell count (14).

Colony formation assay. TE- 1 and KYSE- 150 cells at the logarithmic growth phase were treated with $0.5 \%$ trypsin (Thermo Fisher Scientific, Inc.). After cell counting, 400 cells/well were seeded into a six-well culture plate, with more than three replicates/group. The transfected cells were cultured for 14 days, and the culture medium was changed every 3 days. Following incubation, the cell colonies were fixed with $4 \%$ paraformaldehyde for $20 \mathrm{~min}$ at a room temperature and stained with Giemsa dye (Beyotime Institute of Biotechnology) for $20 \mathrm{~min}$ at a room temperature.

Flow cytometric analysis of apoptosis. Following the corresponding transfections, TE-1 and KYSE-150 cells were 

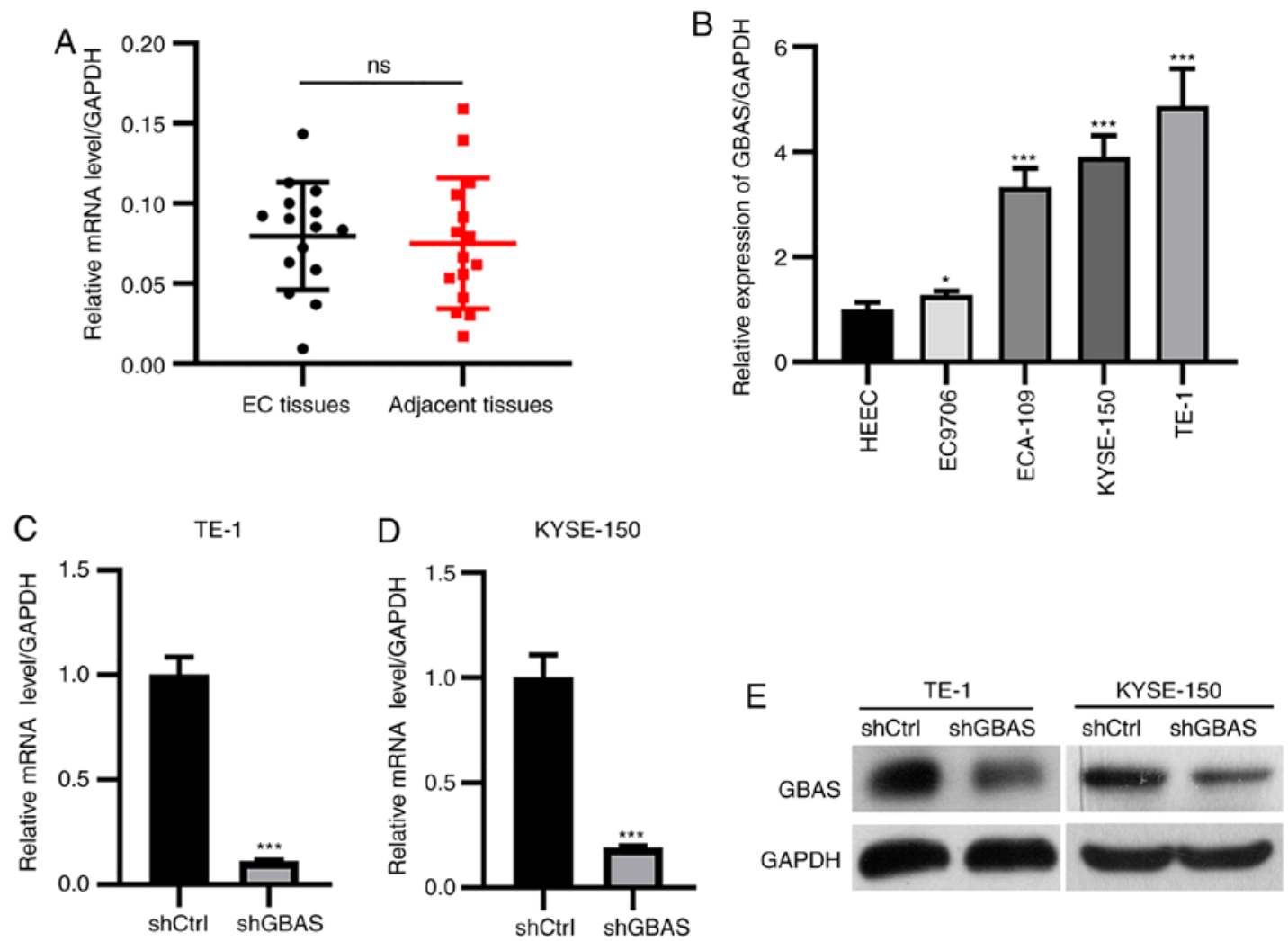

Figure 1. Lentiviral-mediated knockdown of GBAS expression. (A) The expression level of GBAS in patients with EC and adjacent normal tissues detected by RT-qPCR. (B) RT-qPCR was used to analyze mRNA expression levels in the four EC cell lines, TE-1, KYSE-150, ECA-109 and EC9706. GAPDH served as the internal reference control. ${ }^{*} \mathrm{P}<0.05,{ }^{* * *} \mathrm{P}<0.001$ vs. HEEC cells. RT-qPCR was used to confirm knockdown efficency of GBAS in (C) TE-1 and (D) KYSE-150 cells. (E) Western blotting was used to confirm knockdown efficency of GBAS in TE-1 and KYSE-150 cells. GAPDH served as the internal loading control. ${ }^{* * *} \mathrm{P}<0.001$ vs. shCtrl. Ns, no significance; GBAS, glioblastoma-amplified sequence; RT-qPCR, reverse transcription-quantitative PCR; EC, esophageal cancer; sh, short hairpin RNA; Ctrl, control.

digested with trypsin and collected. The cell pellet was washed with ice-cold PBS and then resuspended in $200 \mu \mathrm{l}$ $1 \mathrm{X}$ binding buffer. The cells were subsequently incubated with $10 \mu \mathrm{l}$ Annexin V-allophycocyanin (Beyotime Institute of Technology) at room temperature for $15 \mathrm{~min}$ in the dark. All samples were detected and analyzed using a BD Accuri ${ }^{\text {тм }}$ C6 Plus flow cytometer (BD Biosciences). The apoptosis (early + late apoptosis) were quantified and analyzed using a BD Accuri C6 Software (BD Biosciences).

Cell cycle analysis. Flow cytometry was performed to determine the cell cycle distribution. Briefly, following transfection, TE-1 and KYSE-150 cells were fixed with pre-cooled $75 \%$ ethanol and incubated at $4^{\circ} \mathrm{C}$ for $1 \mathrm{~h}$. The cells were subsequently washed once with cold PBS and centrifuged at $100 \mathrm{xg}$ for $5 \mathrm{~min}$ at $4^{\circ} \mathrm{C}$. The cell pellets were resuspended in propidium iodide (cat. no. P4170; Sigma-Aldrich; Merck KGaA), RNase (cat. no. EN0531; Thermo Fisher Scientific, Inc.), PBS and Triton X-100 (cat. no. 9002-93-1; Sigma-Aldrich; Merck $\mathrm{KGaA}$ ) at a ratio of 25:10:1,000:40. All samples were detected and analyzed using a BD Accuri ${ }^{\mathrm{TM}}$ C6 Plus flow cytometer (BD Biosciences). The distribution of EC cells in each stage of the cell cycle was analyzed using FCS Express IVD software (version 4; De Novo Software).

Statistical analysis. Statistical analysis was performed using SPSS 25.0 software (IBM Corp.) and GraphPad Prism version 8.0 (GraphPad Software, Inc.). The data are presented as the mean \pm SD of three independent repeats. Statistical differences between two groups were determined using an unpaired Student's t-test for unpaired data, or a paired Student's t-test for paired data and statistical differences among multiple groups were analyzed using one way-ANOVA followed by a Tukey's post hoc test. $\mathrm{P}<0.05$ was considered to indicate a statistically significant difference.

\section{Results}

GBAS expression knockdown by lentivirus-mediated transfection. To analyze the expression levels of GBAS in patients with EC, EC clinical tissues were obtained and the expression levels of GBAS were determined using RT-qPCR. Baseline clinicopathological characteristics of the esophageal squamous cell carcinoma are shown in Table S1. The results revealed that there was no significant difference in the expression of GBAS in EC tissues compared with those in normal adjacent tissues (Fig. 1A). The mRNA expression levels of GBAS were subsequently analyzed in normal HEEC cells and four EC cell lines (TE-1, KYSE-150, ECA109 and EC9706) using RT-qPCR. The results demonstrated that the expression levels of GBAS were significantly upregulated in TE-1, KYSE-150, ECA109 and EC9706 cells compared with those in HEEC cells (Fig. 1B). TE-1 and KYSE-150 cells were selected for use in the following experiments due to the high expression 

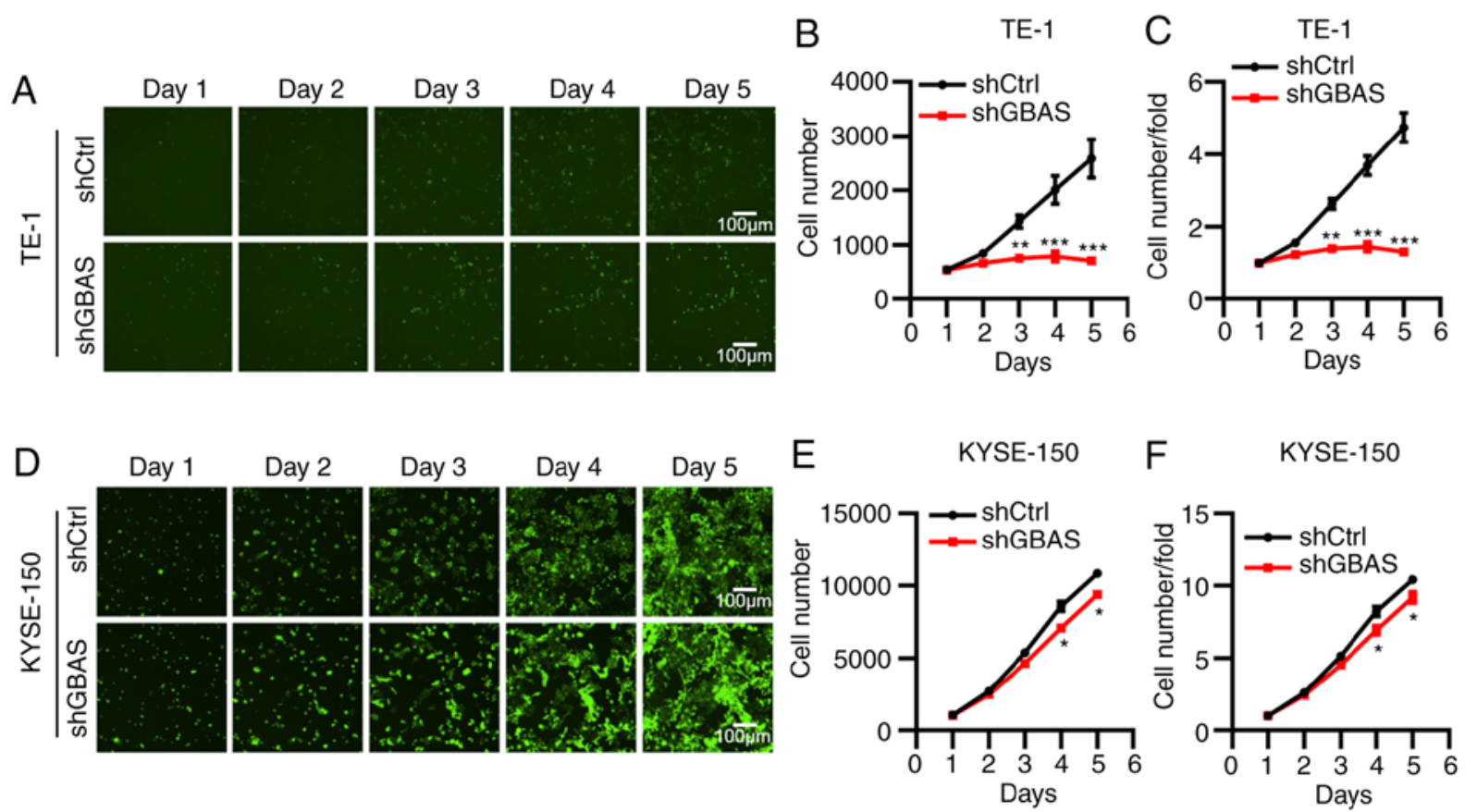

Figure 2. Knockdown of GBAS suppresses the viability of esophageal cancer cells. (A) Celigo image cytometer system was used to determine the viability of TE-1 cells transfected with shGBAS or shCtrl lentiviruses. Scale bar, $100 \mu \mathrm{m}$. (B and C) Statistical analysis of the cell viability assay using TE-1 cells. (D) Celigo image cytometer system was used to determine the viability of KYSE-150 cells transfected with shGBAS or shCtrl lentiviruses. Scale bar, $100 \mu \mathrm{m}$. (E and F) Statistical analysis of cell viability assay using KYSE-150 cells. ${ }^{*} \mathrm{P}<0.05,{ }^{* * *} \mathrm{P}<0.01,{ }^{* * * *} \mathrm{P}<0.001$ vs. shCtrl. GBAS, glioblastoma-amplified sequence; sh, short hairpin RNA; Ctrl, control.

levels of GBAS. The lentiviral-mediated knockdown efficiency of GBAS was verified at both the mRNA and protein levels. RT-qPCR analysis demonstrated that the mRNA expression levels of GBAS were significantly downregulated in both TE-1 and KYSE-150 cells (Fig. 1C and D). Consistent with these findings, the protein expression levels of GBAS were also downregulated in shGBAS-transfected TE-1 and shGBAS-transfected KYSE-150 cells (Fig. 1E). These results suggested that GBAS expression was successfully knocked down in both TE-1 and KYSE-150 cells.

Knockdown of GBAS expression suppresses the viability and colony formation of EC cells. To determine the functional roles of GBAS in regulating the development of EC, cell viability was determined using a Celigo image cytometer system. As shown in Fig. 2, knockdown of GBAS significantly inhibited cell viability following culture for 5 days in both TE-1 (Fig. 2A-C) and KYSE-150 (Fig. 2D-F) cells. In addition, compared with TE-1 cells transfected with shCtrl, colony formation was significantly suppressed in TE-1 cells transfected with shGBAS (Fig. 3A). GBAS knockdown also inhibited colony formation in KYSE-150 cells (Fig. 3B). Collectively, these results indicated that the knockdown of GBAS expression may suppress cell viability and colony formation in EC cell lines.

Knockdown of GBAS promotes apoptosis of EC cells. Accumulating evidence has indicated that cell apoptosis predetermines the onset and progression of malignant tumors due to its regulatory role in cell viability and role in maintaining a constant number of cells $(15,16)$. Thus, the present study aimed to investigate the effect of GBAS knockdown on the apoptosis of TE-1 and KYSE-150 cells by Annexin V staining. The knockdown of GBAS significantly increased the percentage of apoptotic TE-1 (Fig. 4A and C) and KYSE-150 (Fig. 4B and D) cells. Accordingly, the expression levels of apoptotic markers, including caspase-3 and cleaved caspase-3, in TE-1 cells were detected using western blotting. As shown in Fig. 4E, the expression levels of caspase-3 and cleaved caspase- 3 were notably upregulated following GBAS knockdown in TE-1 cells. These results suggested that GBAS knockdown may promote the apoptosis of EC cell lines.

Knockdown of GBAS blocks cell cycle progression in EC cells. Whether GBAS was involved in cell cycle regulation was determined using flow cytometry. Indeed, the knockdown of GBAS in both TE-1 and KYSE-150 cells contributed to cell cycle redistribution. Specifically, following GBAS knockdown, the number of TE- 1 and KYSE-150 cells in the $G_{1}$ phase was significantly increased. The number of cells in the $\mathrm{S}$ phases was significantly decreased in TE-1 cells, while the number of cells in the $\mathrm{G}_{2} / \mathrm{M}$ phases was significantly decreased in KYSE-150 cells (Fig. 5A and B). Taken together, these results suggested that the knockdown of GBAS may block cell cycle progression in EC cell lines.

\section{Discussion}

GBAS expression has been reported to be co-amplified alongside EGFR in multiple types of cancer, which is associated with the upregulated expression levels of the protein $(8,17)$. Previous research has shown that GBAS knockdown can regulate OSCC viability and apoptosis through the p53 signaling pathway (12). However, whether GBAS may be involved in the 

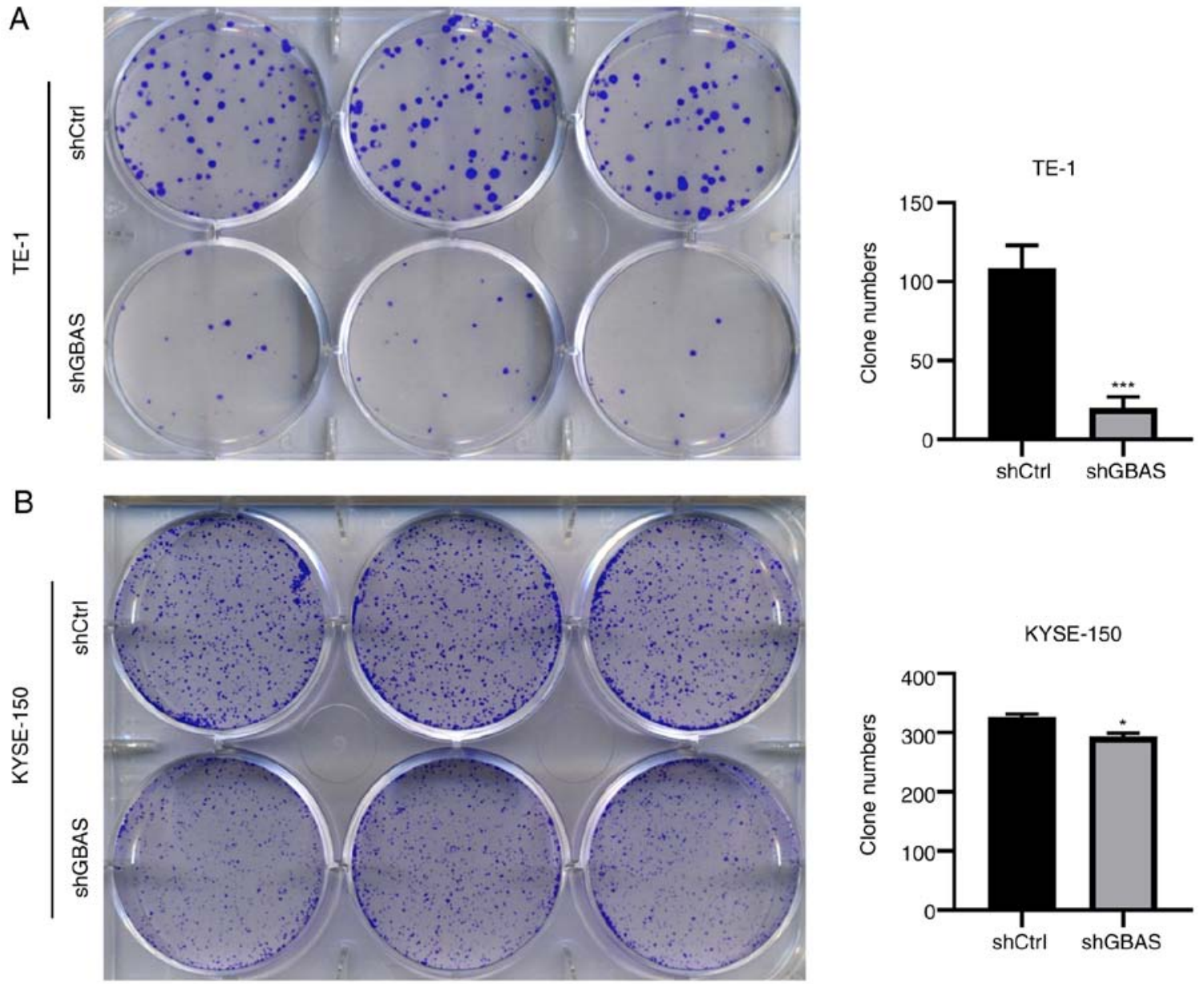

Figure 3. Knockdown of GBAS inhibits colony formation. Colony forming ability of (A) TE-1 and (B) KYSE-150 cells transfected with shGBAS or shCtrl. Left, representative images. Right, quantitative results. ${ }^{*} \mathrm{P}<0.05,{ }^{* * * *} \mathrm{P}<0.001$ vs. shCtrl. GBAS, glioblastoma-amplified sequence; sh, short hairpin RNA; Ctrl, control.

development and progression of solid tumor types, particularly EC, remains unclear. Thus, further investigations are required to elucidate the biological function and underlying molecular mechanisms of GBAS in EC progression. The results of the present study demonstrated that GBAS regulated cell viability, colony formation and cell apoptosis, as well as the cell cycle distribution of EC cells, which in turn may serve important functional roles in the development of EC. To the best of our knowledge, these findings have not been reported before in EC.

To further elucidate the clinical relevance of GBAS expression in EC, the present study analyzed the expression levels of GBAS in EC and adjacent normal tissues. The present study revealed that the expression level of GBAS was not significantly different in EC tissues compared with the adjacent normal tissues. Unfortunately, owing to the limited availability of EC tissues, only 15 paired samples were used in the present analysis. Thus, one of the major limitations in the present study was the very small sample size making it difficult to analyze the association between EC tissues and GBAS expression; more clinical samples need to be collected for further study. Nevertheless, previous studies suggest that the expression of GBAS is dysregulated in several types of malignancies and GBAS expression is co-amplified with EGFR in human gliomas $(8,11,18)$. However, GBAS is downregulated in oral squamous cell carcinoma and involved in controlling proliferation and apoptosis via the p53 signaling pathway (12). Therefore, it was hypothesized that GBAS may be an oncogene associated with EC development. However, the results suggested that GBAS may function as a novel oncogene, although its expression levels were not significantly different in EC tissues compared with normal tissues.

The present study subsequently aimed to elucidate the functional biological roles of GBAS in EC cells via loss-of-function in vitro studies. The results revealed that the knockdown of GBAS could effectively inhibit EC cell viability and colony formation and induce apoptosis. Considering that the dysregulation of cell viability and apoptosis are usually considered as markers of tumorigenesis $(19,20)$, it was hypothesized that the dysregulated expression of GBAS in EC may be associated with the overall survival of patients. Similar findings have also been reported in other types of cancer, including OSCC, bladder cancer and early-stage NSCLC (11). As reported by Hong et al (11), overexpression of GBAS promoted the centrosome amplification rate, as well as cell migration and invasion in bladder cancer cells. In addition, other assays in this previous study revealed that GBAS expression co-amplified with a variant of EGFR, rs9642391C $>\mathrm{G}$, was associated with survival outcomes in patients with early-stage NSCLC (11). Another previous study suggested that GBAS expression was upregulated in OSCC, and knockdown of GBAS could regulate OSCC cell growth and apoptosis via activating the p53 signaling pathway (12). However, as the viability and 

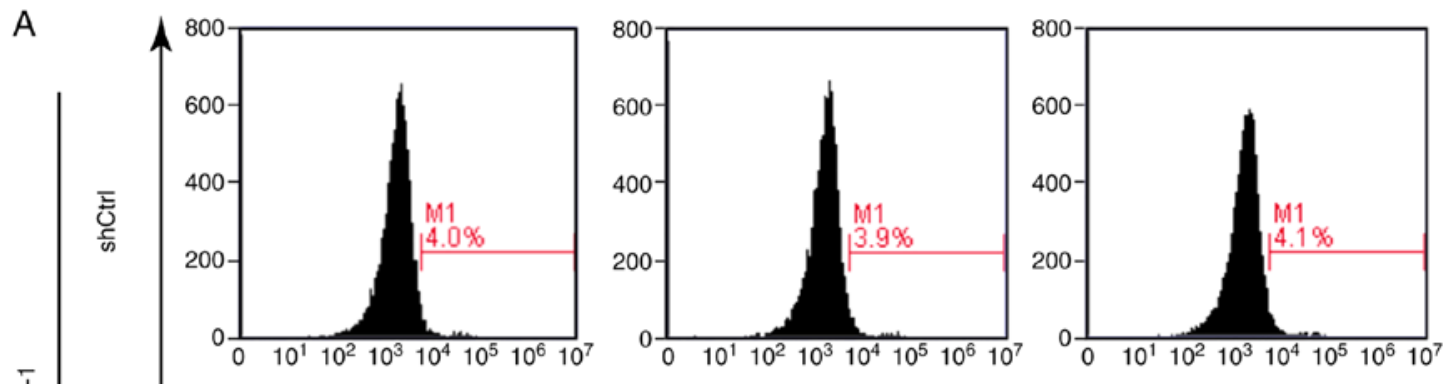

荘
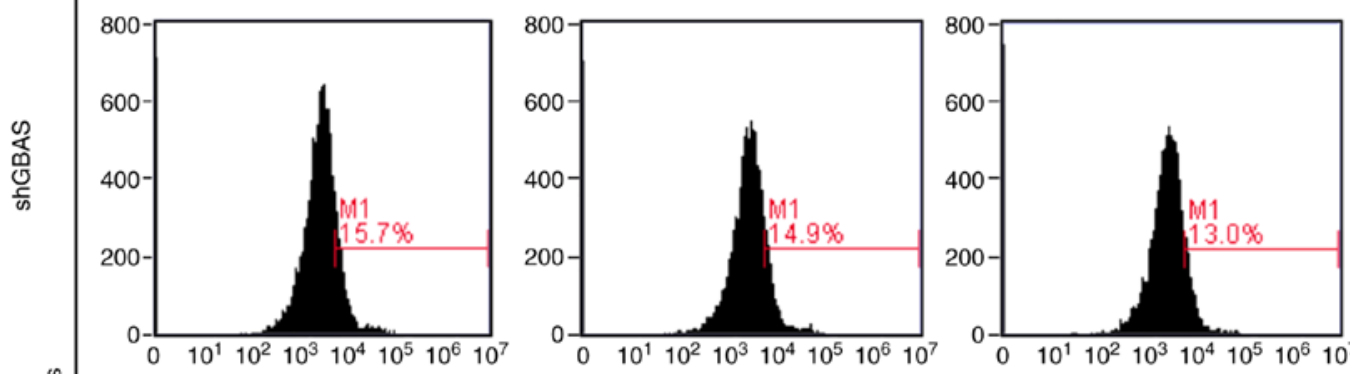

B

亭
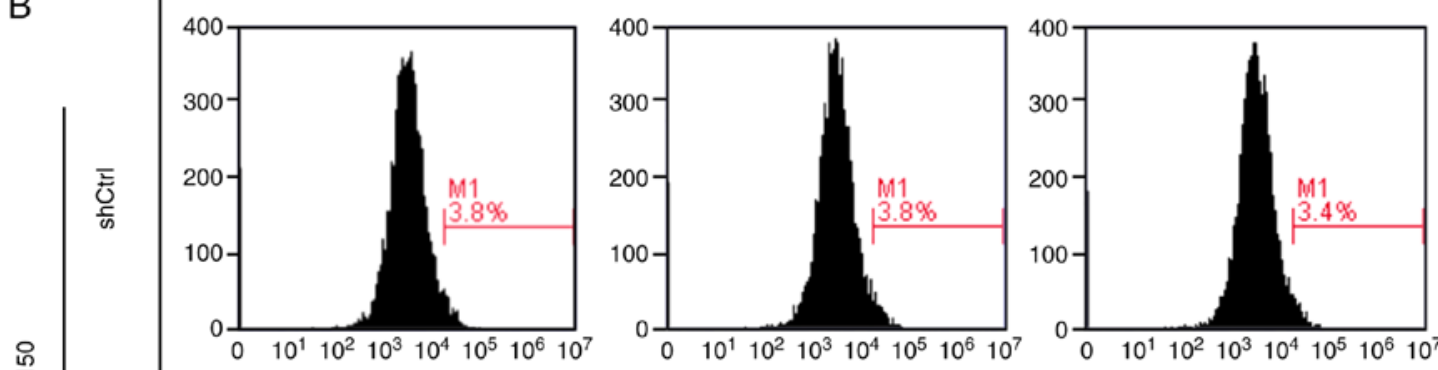

菂
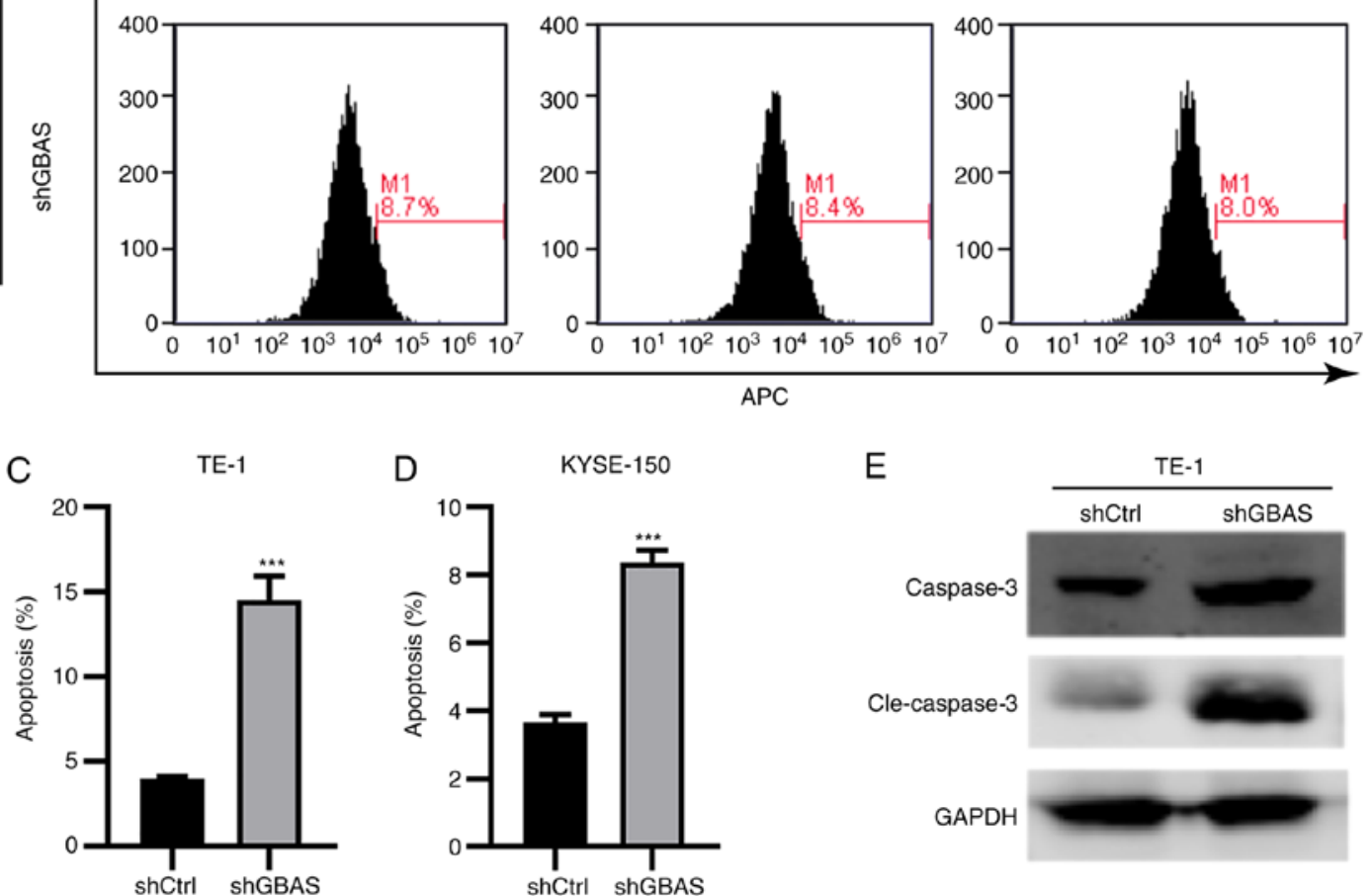

Figure 4. Knockdown of GBAS promotes the apoptosis of esophageal cancer cells. Cell apoptosis was determined by Annexin V staining in (A) TE-1 and (B) KYSE-150 cells transfected with shGBAS or shCtrl. The statistical analysis of (C) TE-1 and (D) KYSE-150 are presented. ${ }^{* * *}$ P $<0.001$ vs. shCtrl. (E) Western blotting was used to detect the protein expression of caspase-3 and Cle-caspase-3 in the shCtrl and shGBAS groups in TE-1 cells. GAPDH served as the internal loading control. GBAS, glioblastoma-amplified sequence; sh, short hairpin RNA; Ctrl, control; Cle-, cleaved.

proliferative ability of EC cells following overexpression of GBAS were not investigated in the present study, this will be further investigated in future studies.
In addition to the determined functional roles of GBAS in cell viability and apoptosis, the results of the flow cytometric analysis revealed that the knockdown of GBAS 

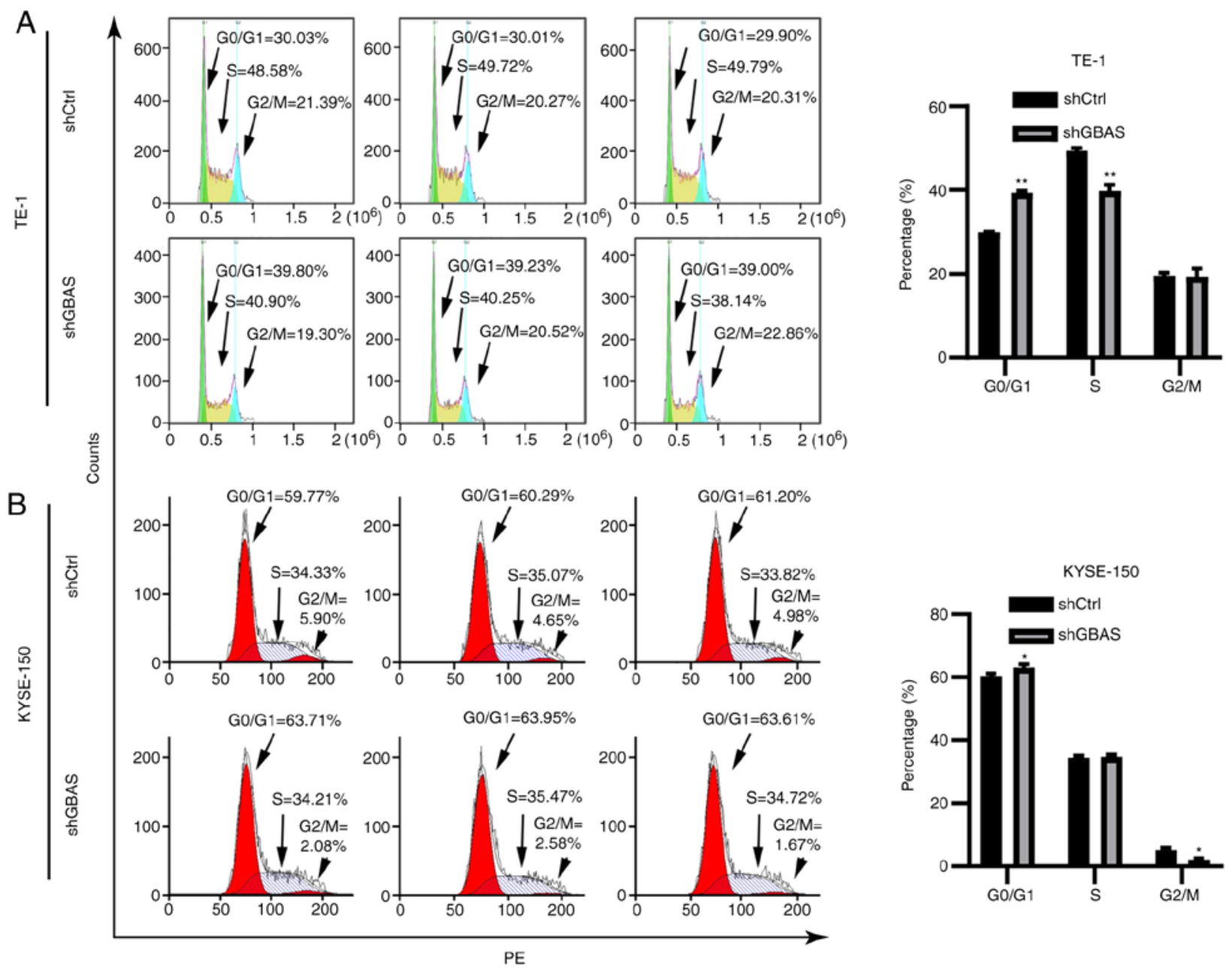

Figure 5. Knockdown of GBAS blocks the cell cycle progression of esophageal cancer cells. Flow cytometry was used to determine the cell cycle distirbution of (A) TE-1 and (B) KYSE-150 cells transfected with shGBAS or shCtrl. Left, representative images. Right, quantitative results. ${ }^{*} \mathrm{P}<0.05$, ${ }^{* *} \mathrm{P}<0.01$ vs. shCtrl. GBAS, glioblastoma-amplified sequence; sh, short hairpin RNA; Ctrl, control.

altered the cell cycle distribution of EC cells. Notably, GBAS knockdown arrested the cell cycle at the $\mathrm{G}_{1}$ phase, which suggested that GBAS may regulate the development of EC through arresting the cell cycle. Of note, the role of GBAS in EC is consistent with the previously reported roles of other members of the NIPSNAP family (21-23). However, the mechanism underlying the GBAS-induced regulation of the EC cell cycle still remains poorly understood. The present found that following GBAS knockdown, the results among two EC cell lines were not consistent, which might be owing to the difference in the characteristics and culture conditions of the two cell lines.

In conclusion, the findings of the present study suggested that GBAS may promote the progression and development of EC by regulating cell viability, apoptosis and cell cycle progression, suggesting that GBAS may play a role in EC.

\section{Acknowledgements}

Not applicable.

\section{Funding}

No funding was received.

\section{Availability of data and materials}

The datasets used and/or analyzed during the current study are available from the corresponding author on reasonable request.

\section{Authors' contributions}

JP and JTH performed the experiments and designed the study. JP and KM conceptualized the study and performed the experiments. HR, BX and JZ analyzed and interpreted the data. JP and JTH drafted and revised the manuscript. JP and JTH confirm the authenticity of all the raw data. All authors read and approved the final manuscript.

\section{Ethics approval and consent to participate}

The present study protocol was approved by the ethics committee of Sichuan Cancer Hospital (Sichuan, China) and written informed consent was obtained from all patients prior to participation.

\section{Patient consent for publication}

Not applicable. 


\section{Competing interests}

The authors declare that they have no competing interests.

\section{References}

1. Hagymasi K and Tulassay Z: Risk factors for esophageal cancer, and possible genetic background. Orv Hetil 150: 407-413, 2009 (In Hungarian).

2. Tsuyama S, Kohsaka S, Hayashi T, Suehara Y, Hashimoto T, Kajiyama Y, Tsurumaru M, Ueno T, Mano H, Yao T and Saito T: Comprehensive clinicopathological and molecular analysis of primary malignant melanoma of the oesophagus. Histopathology 78: 240-251, 2021.

3. Chen R, Zheng RS, Zhang SW, Zeng HM, Wang SM, Sun KX, Gu XY, Wei WW and He J: Analysis of incidence and mortality of esophageal cancer in China, 2015. Zhonghua Yu Fang Yi Xue Za Zhi 53: 1094-1097, 2019 (In Chinese).

4. Cools-Lartigue J, Spicer J and Ferri LE: Current status of management of malignant disease: Current management of esophageal cancer. J Gastrointest Surg 19: 964-972, 2015.

5. van Laarhoven HW: Is chemotherapy for advanced or metastatic oesophageal squamous cell carcinoma no longer needed? Lancet Oncol 21: 743-745, 2020.

6. Fatehi Hassanabad A, Chehade R, Breadner D and Raphael J: Esophageal carcinoma: Towards targeted therapies. Cell Oncol (Dordr) 43: 195-209, 2020.

7. Seroussi E, Pan HQ, Kedra D, Roe BA and Dumanski JP: Characterization of the human NIPSNAP1 gene from 22q12: A member of a novel gene family. Gene 212: 13-20, 1998 .

8. Wang XY, Smith DI, Liu W and James CD: GBAS, a novel gene encoding a protein with tyrosine phosphorylation sites and a transmembrane domain, is co-amplified with EGFR. Genomics 49: 448-451, 1998.

9. Abudu YP, Pankiv S, Mathai BJ, Lamark T, Johansen T and Simonsen A: NIPSNAP1 and NIPSNAP2 act as 'eat me' signals to allow sustained recruitment of autophagy receptors during mitophagy. Autophagy 15: 1845-1847, 2019.

10. Abudu YP, Pankiv S, Mathai BJ, Håkon Lystad A, Bindesbøll C, Brenne HB, Yoke Wui Ng M, Thiede B, Yamamoto A, Mutugi Nthiga T, et al: NIPSNAP1 and NIPSNAP2 Act as 'Eat Me' signals for mitophagy. Dev Cell 49: 509-525.e12, 2019.

11. Hong MJ, Lee SY, Choi JE, Kang HG, Do SK, Lee JH, Yoo SS, Lee EB, Seok Y, Cho S, et al: Intronic variant of EGFR is associated with GBAS expression and survival outcome of early-stage non-small cell lung cancer. Thorac Cancer 9: 916-923, 2018.
12. Wang X, Bai Y, Han Y, Meng J and Liu H: Downregulation of GBAS regulates oral squamous cell carcinoma proliferation and apoptosis via the p53 signaling pathway. Onco Targets Ther 12: 3729-3742, 2019.

13. Livak KJ and Schmittgen TD: Analysis of relative gene expression data using real-time quantitative PCR and the 2(-Delta Delta C(T)) method. Methods 25: 402-408, 2001.

14. Wang L and Ouyang L: Effects of EIF3B gene downregulation on apoptosis and proliferation of human ovarian cancer SKOV3 and HO-8910 cells. Biomed Pharmacother 109: 831-837, 2019.

15. Green DR: Cancer and apoptosis: Who is built to last? Cancer Cell 31: 2-4, 2017.

16. Kaczanowski S: Apoptosis: Its origin, history, maintenance and the medical implications for cancer and aging. Phys Biol 13: 031001, 2016.

17. Cheng LS, Davis RC, Raffel LJ, Xiang AH, Wang N, Quiñones M, Wen PZ, Toscano E, Diaz J, Pressman S, et al: Coincident linkage of fasting plasma insulin and blood pressure to chromosome $7 \mathrm{q}$ in hypertensive hispanic families. Circulation 104: 1255-1260, 2001 .

18. Wang X, Li S and Liu H: Co-delivery of chitosan nanoparticles of 5-aminolevulinic acid and shGBAS for improving photodynamic therapy efficacy in oral squamous cell carcinomas. Photodiagnosis Photodyn Ther 34: 102218, 2021.

19. Gregory CD, Ford CA and Voss JJ: Microenvironmental effects of cell death in malignant disease. Adv Exp Med Biol 930: 51-88, 2016.

20. Pachl J, Duska F, Waldauf P, Fric M, Fanta J and Zdarsky E: Apoptosis as an early event in the development of multiple organ failure? Physiol Res 54: 697-699, 2005.

21. Malhotra A, Shibata Y, Hall IM and Dutta A: Chromosomal structural variations during progression of a prostate epithelial cell line to a malignant metastatic state inactivate the NF2, NIPSNAP1, UGT2B17, and LPIN2 genes. Cancer Biol Ther 14: 840-852, 2013.

22. Schoeber JP, Topala CN, Lee KP, Lambers TT, Ricard G, van der Kemp AW, Huynen MA, Hoenderop JG and Bindels RJ: Identification of Nipsnapl as a novel auxiliary protein inhibiting TRPV6 activity. Pflugers Arch 457: 91-101, 2008.

23. Okuda-Ashitaka E, Minami T, Tsubouchi S, Kiyonari $H$, Iwamatsu A, Noda T, Handa $\mathrm{H}$ and Ito S: Identification of NIPSNAP1 as a nocistatin-interacting protein involving pain transmission. J Biol Chem 287: 10403-10413, 2012.

This work is licensed under a Creative Commons Attribution-NonCommercial-NoDerivatives 4.0 International (CC BY-NC-ND 4.0) License. 